\title{
Effects of Dihydroartemisinin, a Metabolite of Artemisinin, on Colon Cancer Chemoprevention and Adaptive Immune Regulation
}

Chong-Zhi Wang ( $\square$ czwang@dacc.uchicago.edu)

University of Chicago Department of Anesthesia and Critical Care https://orcid.org/0000-0002-09502109

Chunping Wan

Yunnan University of Traditional Chinese Medicine

Yun Luo

University of Chicago Department of Anesthesia and Critical Care

Chun-Feng Zhang

China Pharmaceutical University

Qi-Hui Zhang

Chongqing University

Lina Chen

Nanjing Medical University

Zhi Liu

University of Chicago Department of Anesthesia and Critical Care

Daniel Henry Wang

University of Chicago Department of Anesthesia and Critical Care

Mallory Lager

University of Chicago Department of Anesthesia and Critical Care

Cang-Hai Li

China Academy of Chinese Medical Sciences Institute of Chinese Materia Medica

Ting-Liang Jiang

China Academy of Chinese Medical Sciences Institute of Chinese Materia Medica

Lifei Hou

Harvard Medical School

Chun-Su Yuan

University of Chicago Department of Anesthesia and Critical Care

Research Article 
Keywords: Dihydroartemisinin, artemisinin, cell cycle and apoptosis, colorectal cancer, $T$ helper 17 cells, anti-inflammation and adaptive immunity

Posted Date: August 20th, 2021

DOI: https://doi.org/10.21203/rs.3.rs-744220/v1

License: (c) (1) This work is licensed under a Creative Commons Attribution 4.0 International License. Read Full License

Version of Record: A version of this preprint was published at Molecular Biology Reports on January 18th, 2022. See the published version at https://doi.org/10.1007/s11033-021-07079-1. 


\section{Abstract}

Artemisinin (ART) is an anti-malaria natural compound with a moderate anticancer action. As a metabolite of ART, dihydroartemisinin (DHA) may have stronger anti-colorectal cancer (CRC) bioactivities. However, the effects of DHA and ART on CRC chemoprevention have not been systematically evaluated and compared, including on adaptive immune regulation. Coupled with a newly-established HPLC analytical method, enteric microbiome biotransformation was conducted to identify if the DHA is a gut microbial metabolite of ART. The anti-CRC potential of these compounds was compared using two different human CRC cell lines for cell cycle arrest, apoptotic induction, and anti-inflammation activities. Naive $\mathrm{CD} 4^{+} \mathrm{T}$ cells were also obtained for testing the compounds on the differentiation of Treg, Th1 and Th17. Using compound extraction and analytical methods, we observed for the first time that ART completely converted into its metabolites within $24 \mathrm{~h}$, but no DHA was detected. Although ART did not obviously influence cancer cell growth in the concentration tested, DHA very significantly inhibited the cancer cell growth at relatively low concentrations. DHA included G2/M cell cycle arrest via upregulation of cyclin A and apoptosis. Both ART and DHA downregulated the pro-inflammatory cytokine expression. The DHA significantly promoted Treg cell proliferation, while both ART and DHA inhibited Th1 and Th17 cell differentiation. As a metabolite of ART, DHA possessed stronger anti-CRC activities. The DHA significantly inhibited cell growth via cell cycle arrest, apoptosis induction and anti-inflammation actions. The adaptive immune regulation is a related mechanism of actions for the observed effects.

\section{Introduction}

Artemisia annua L. or artemisia, is an herb traditionally used in Chinese medicine to treat fever, inflammation, and malaria infections [1]. One of its active compounds, artemisinin, a sesquiterpene trioxane lactone agent, was identified by Chinese scientists in 1970s. This novel compound has been used in combination with other drugs to treat drug-resistant malaria and has saved millions of lives. The 2015 Nobel Prize in Physiology or Medicine was awarded to Professor Youyou Tu for her key contributions to the discovery of artemisinin (ART) [2,3]. Afterwards, Professor Tu discovered dihydroartemisinin (DHA) that greatly improved ART's water solubility and treatment efficacy against malaria [4].

Any given botanical is likely consisted of many chemically known or unknown single constituents, and some of the constituents could be pharmacologically categorized as bioactive compounds. Meanwhile, medicinal herbs and their derivatives, including ART, are almost always taken orally. After intake, their parent compounds could be biotransformed by enteric microbiome, and the metabolites could possess even higher bioactivities compared with the parent ones [5]. Studies have shown that after oral ART administration, DHA can be detected in plasma, suggesting the latter is a metabolite of the former $[6,7]$. However, after ART oral ingestion, whether the parent compound ART is metabolized by the enteric microbiome into DHA has not been examined. 
When botanical-origin novel compounds introduced for certain medical treatment in humans, researchers often start to look for their additional therapeutic capabilities, especially from the herb's original and empirical indications, such as using artemisia against inflammation. Consequently, both ART and DHA have been used to evaluate their effects on inflammatory bowel disease and variable activities have been reported [8-10].

Previous data indicated that inflammatory bowel disease (IBD), including ulcerative colitis and Crohn's disease, is a risk factor for colon cancer initiation and development [11, 12]. Epidemiological, experimental, and clinical studies provide evidence that anti-inflammatory phytochemicals possess unique modes of action for body's homeostasis to prevent the disease and its progression [13-16]. Interestingly, salicylate, the active metabolite of aspirin, and the antidiabetic drug metformin are both originally derived from botanicals, which activate the energy sensing kinase AMPK and show antiinflammation and anticancer effects; while ART and its analogs also activate the AMPK pathway $[17,18]$.

Artemisia has been reported to possess both anti-inflammation and anti-cancer actions with a moderate efficacy against colorectal cancer (CRC) [19]. With its established safety record in millions of malarial patients, ART is also being investigated in diseases like infections, inflammation, and cancers [20]. Due to its relatively low anticancer potential, the perspective of cancer chemoprevention of ART is restricted. However, the effects of DHA, a metabolite of ART, on CRC chemoprevention have not been systematically evaluated especially in comparison with the ART. In this study, using a newly-established HPLC analytical method, enteric microbiome biotransformation will be conducted to identify if the DHA is a gut microbial metabolite of ART. Then, the anti-CRC potential of the two compounds will be compared using two different human colon cancer cell lines for cell cycle arrest and induction of apoptosis. Further, their mechanisms of action in relation to the anti-inflammation and adaptive immune regulation will be examined.

\section{Materials And Methods}

\section{Reagents}

Artemisinin and dihydroartemisinin are obtained from Sigma-Aldrich (St. Louis, MO). The chemicals and solvents used were all for high performance liquid chromatography or HPLC grade. Plasticware was purchased from Falcon Labware (Franklin Lakes, NJ). Test media, insulin trypsin, glutamine, and buffered saline were purchased from Mediatech (Herndon, VA). Antibiotics, such as streptomycin and penicillin, were purchased from Sigma-Aldrich (St. Louis, MO). Fetal bovine serum (FBS) was purchased from Life Technologies Corporation (Grand Island, NY). For cell proliferation study, the MTS kit was purchased from Promega (Madison, WI). Cell cycle staining buffer was purchased from Biosciences Pharmingen (San Diego, CA), and cell apoptosis detection kit was purchased from Biosciences (Rockville, MD).

\section{Biotransformation and sample processing}


The human fecal specimens were collected from five healthy adult male American subjects (no smoking and alcohol, no current medication, no use of antibiotics) and stored in a $-85^{\circ} \mathrm{C}$ freezer. The fecal specimens were mixed, and the mixture was suspended well in phosphate buffer to obtain a fecal slurry. The slurry was filtered through muslin to remove particulate material. The resulting fecal slurry was used as intestinal microbiome fraction. One microliter of the fecal slurry was mixed with $14 \mathrm{ml}$ anaerobic medium containing $3.75 \mathrm{mg}$ of ART. They were anaerobically incubated at $37^{\circ} \mathrm{C}$ for 12 and $24 \mathrm{~h}$. Then, the reaction mixture was extracted with ethyl ether. The ethyl ether layer was collected, and dried in a water bath $\left(37^{\circ} \mathrm{C}\right)$. Then the residue was dissolved in methanol. The methanol solution was centrifuged at $13,000 \times \mathrm{g}$ for $10 \mathrm{~min}$ before HPLC analysis.

\section{HPLC Analysis}

Our laboratory uses a Waters instrument 2965 (Milford, MA) for HPLC analysis. This system (Model 996) was used for constituent peak identification and integration, supported by a Waters Empower software. The separation was carried out on a Prodigy ODS (2) column $(5 \mu \mathrm{m}, 250 \times 3.2 \mathrm{~mm}$ I.D.) (Phenomenex, Torrance, CA) with a guard column (Phenomenex ProdigyTM, $3.0 \times 4.0 \mathrm{~mm}$ I.D.). For HPLC analysis, a 20$\mu \mathrm{l}$ sample was injected into the column and eluted at room temperature with a constant flow rate of 1.0 $\mathrm{ml} / \mathrm{min}$. Acetonitrile (solvent $\mathrm{A}$ ) and water (solvent B) were used as mobile phase. Gradient elution started with $40 \%$ solvent $A$ and $60 \%$ solvent $B$, changed to $60 \%$ A for 10 min and held for 2 min, then changed to $90 \%$ A for $3 \mathrm{~min}$ and held for $3 \mathrm{~min}$. Finally, it was changed to $40 \%$ A for $4 \mathrm{~min}$ and held for $3 \mathrm{~min}$ to rebalance the separated column. The detection wavelength was set to $210 \mathrm{~nm}$.

\section{Cancer Cell Lines and Cell Culture}

HT-29 and HCT-116 human colon cancer cells were purchased from American Type Culture Collection (Manassas, VA). These cells were grown in McCoy's 5A medium with 10\% FBS and 50 IU streptomycinpenicillin in the humidified air at $37^{\circ} \mathrm{C}$ with $5 \% \mathrm{CO}_{2}$.

\section{Cell Proliferation Analysis}

The solvent used to dissolve ART and DHA were DMSO. The 96-well plates were used to seed cells and test response to drug treatment. Drug treatment durations were 24,48 , or $72 \mathrm{~h}$. MTS assay was employed to determine cell proliferation. As our previous publications, absorbance was recorded at $490 \mathrm{~nm}[21,22]$.

\section{Cell Cycle and Cyclin A Assays}

HCT-116 cells were seeded on 24-well plates. On day 2, the medium was changed, during which different ART and DHA concentrations were used to treat the cells. After $48 \mathrm{~h}$, the cells were harvested and fixed with $80 \%$ ethanol. After $0.25 \%$ Triton X-100 treatment, the cells were resuspended in PBS containing PI, RNase, and cyclin A-FITC. FACScan flow cytometer (Becton Dickinson, Mountain View, CA) and FlowJo 10.7.1 software (Tree Star, Ashland, OR) were used to assay cell cycle and cyclin A. At least 10,000 cells were recorded for each assay. 


\section{Apoptosis Analysis}

HCT-116 cells were seeded on 24-well plates. On day 2, the medium was changed, during which different ART and DHA concentrations were used to treat the cells. After $48 \mathrm{~h}$, both floating and adherent cells were harvested. The cells were stained with PI and annexin V. FACScan flow cytometer were used to assay apoptotic cells. At least 20,000 cells were recorded for each assay.

\section{IL-8 secretion assay}

Using 24-well plates, HT-29 cells were cultured for $48 \mathrm{~h}$. Fresh medium containing lipopolysaccharide (LPS, $100 \mathrm{ng} / \mathrm{ml}$ ) was added, which served as a control. Different concentrations of ART/DHA and LPS added in the medium were set as the experimental group. After incubating for $6 \mathrm{~h}$, the secreted IL-8 in the medium was determined by enzyme-linked immunosorbent assay.

\section{Naive CD4 Cell Isolation and Differentiation of T-helper Cells}

Single cell suspensions were prepared by using 4-6 wk old naive C57BL/6 (B6) mice's spleens. Pooled splenocytes (after erythrocyte lysis) were depleted of CD11b+, CD8a+, and CD19+ cells using biotinylated primary antibodies (BioLegend, San Diego, CA, USA) and streptavidin-coated secondary magnetic particles (Stem Cell Technologies, Vancouver, BC, Canada). T cell differentiation was based on our previous publications $[23,24]$. Test compounds were initially added into the culture medium. After 3 days, the differentiated cells were harvested, then in the presence of Brefeldin A, the cells were restimulated for $4 \mathrm{~h}$ with PMA (50 $\mathrm{ng} / \mathrm{ml})$ and ionomycin $(750 \mathrm{ng} / \mathrm{ml})$ (Sigma-Aldrich).

\section{Statistical Analysis}

Data were expressed as mean $\pm \mathrm{SE}$. In general, a one-way ANOVA was used to define the data significant level. In some instances, Student's t-test was employed for two-group comparison. In all cases, $\mathrm{P}<0.05$ was considered as statistically significant.

\section{Results}

\section{HPLC Analysis of Purities of ART and DHA}

The chemical structures of ART and DHA are shown in Fig. 1A. An analytical method was developed that determined these ART and DHA simultaneously using a reverse phase column with gradient elution. To date, almost all reports on the HPLC analysis of ART and DHA employed reverse phase column with an isocratic mobile phase of acetonitrile:water (60:40) [25-27]. Using our reverse phase column with the isocratic mobile elution, the retention time for ART was $4.904 \mathrm{~min}$, while for DHA were 4.202 and 4.869 min. Although the two peaks of DHAa and DHAß can be separated well, in this condition, ART cannot be separated with DHA . Thus, the reported method has significant defects in simultaneous determination of ART and DHA. 
To increase the resolution of separation, in some reports, buffers or acids are used in these elution systems. However, the buffer cannot be stored in the column for a long time, and a complex, timeconsuming process has to be conducted each time the assay is complete. To avoid buffer, acetonitrile and water, two simple, easily prepared eluents that are safe for the column, were used. In this study, a gradient elution program was developed without buffer to separate ART and DHA. As shown in Fig. 1B, using our method, the retention time (Rt) for ART are $10.566 \mathrm{~min}$, while Rt for DHAa and DHA $\beta$ are 6.671 and $8.905 \mathrm{~min}$. Those three peaks are baseline separated. The purity of ART and DHA are $98.7 \%$ and $98.5 \%$, respectively. The contents of DHAa and DHA $\beta$ in DHA are $70.2 \%$ and $28.2 \%$, respectively, and the ratio for $\alpha$ and $\beta$ form of DHA is 2.49:1.

\section{Gut Microbiome Biotransformation of ART}

Based on previous reports, DHA is an in vivo metabolite of ART [6,7]. Like many other herbal medicines, Artemisia annua is taken orally. After oral ingestion, the main constituent artemisinin is exposed to gut microflora in the gastrointestinal tract. Whether DHA is converted by the gut microbiome or by enzymes in vivo from ART is still unknown. Since there is no enteric microbial converts report on ART, gut microbiome biotransformation tests were performed on ART.

As shown in Fig. 1C, for the control groups at 0,12 and $24 \mathrm{~h}$, since there is no peak at the retention times of ART and DHA, the background of gut microbiome did not influence the determination of ART and DHA. When ART incubated with gut microbiome for $12 \mathrm{~h}$, compared to the chromatogram at $0 \mathrm{~h}$, the peak height of ART was reduced, suggesting ART was transformed by gut microbiome to its metabolites, and two new peaks were detected. After $24 \mathrm{~h}$ incubation, ART was not detected, suggesting ART was absolutely transformed to its metabolites in $24 \mathrm{~h}$, while three new peaks were detected. UV spectra of those three peaks suggested that the compound at Rt $7.823 \mathrm{~min}$ is likely a metabolite of ART. Because UV spectra of other two peaks are much different to ART or DHA, those two peaks should be produced by the gut microbiome, which was supported by the chromatogram from control group, in which a compound at Rt 11.116 min was detected. Our data firstly proved that DHA is not a gut microbial metabolite of ART.

\section{Antiproliferative Effects of ART and DHA}

To evaluate anti-proliferative effects of ART and DHA, a two-step-strategy was emplyed. In the first step, one time point was investigated with relatively large intervals in compound concentration. As shown in Fig. 2A, for the cell line HCT-116, DHA showed significant antiproliferative effect in concentrations of $>10$ $\mu \mathrm{M}(\mathrm{p}<0.05, p<0.01)$. Morphological observation was performed after crystal violet staining, showing that ART did not influence HCT-116 cell growth, while DHA obviously inhibited cell growth at 15 and $25 \mu \mathrm{M}$ (Fig. 2B). Similar effects were observed in HT-29 cell line, but the active concentration of DHA was $30 \mu \mathrm{M}$, which is higher than that on HCT-116 cells.

Due to DHA showing strong inhibition activities on HCT-116 cells, this cell line was employed for further studies. In the second step, the time- and dose-dependent effects were investigated. Cell proliferations were observed at 24,48 , and $72 \mathrm{~h}$, and more precise concentration ranges were employed. The treatment 
concentrations for both compounds were 5-25 $\mu \mathrm{M}$. As shown in Fig. 2C, after treatment with DHA, by doses over $10 \mu \mathrm{M}$, dose- and time-dependent antiproliferative effects were observed. However, ART did not inhibit cancer cell growth (Fig. 2C).

\section{ART and DHA on Cancer Cell Cycle}

Based on MTS results, DHA showed significant antiproliferation effects on HCT-116 cells with the doses over $10 \mu \mathrm{M}$. Thus, concentrations used in cell cycle tests were of $10-25 \mu \mathrm{M}$ for $48 \mathrm{~h}$. Fig. 3A shows that 10-25 $\mu \mathrm{M}$ of DHA treatment changed cell cycle profiles. At $10 \mu \mathrm{M}$, DHA obviously reduced S-phase proportion and increased G2/M-phase proportion. Treatment with 15-25 $\mu \mathrm{M}$ showed more significant changes. After treatment with $20 \mu \mathrm{M}$ of DHA for $48 \mathrm{~h}$, in comparison to the control $48.5 \%$ of $\mathrm{G} 1,29.5 \%$ of $M$, and $17.9 \%$ of $G 2 / M)$, cells in G1-phase were $32.7 \%$ ( $p<0.01)$, M-phase $25.0 \%(p<0.01)$, and $G 2 / M$ phase $40.8 \%(p<0.01)$. DHA treatment significantly decreased G1- and S-phase cells and increased G2/M-phase cells. However, cell cycle effects of ART in HCT-116 cells were not found (Fig. 3B).

\section{Effects ART and DHA on Expression of cyclin A}

Cell cycle progression is regulated by cyclins, particularly cyclin A for the S-phase and passage through the G2/M-phase. The observation of G2/M phase cancer cell cycle arrest through GBC led to further investigation of cell cycle regulation through evaluating the expression of cyclin A. Using HCT-116 cells, for the control group, the cell proportion for cyclin A positive cells was $14.7 \%$. After $48 \mathrm{~h}$ treatment with $10,15,20$, and $25 \mu \mathrm{M}$ of DHA, the cyclin A positive proportions increased to $33.3 \%, 38.3 \%, 44.1 \%$, and $51.0 \%$, respectively (all $p<0.01$ ) (Fig. 3B). On the other hand, with ART treatment at same concentrations, the expression of cyclin A was not changed. Therefore, DHA treatment induced an obvious increase in cyclin A expression on HCT-116 cells.

\section{Apoptotic Induction of ART and DHA on Colorectal Cancer Cells}

To further investigate mechanisms of DHA's cell growth inhibition, an apoptotic assay was conducted within the staining of PI and annexin V. For PI and annexin V staining, negative for both are viable cells, while positive for both are late apoptotic or necrotic cells. PI-positive and annexin V-negative are cells that underwent necrosis, while annexin V-positive and PI-negative are early apoptotic cells. As shown in Fig. $4 \mathrm{~A}$, the early and late apoptotic cells for the control were $5.3 \%$ and $3.4 \%$, respectively. Treatments with DHA at 20-30 $\mu \mathrm{M}$ increased apoptotic cells significantly. For example, $30 \mu \mathrm{g} / \mathrm{ml}$ of DHA treatment for $48 \mathrm{~h}$ resulted in an increasing of early and late apoptotic cells to $14.5 \%$ and $26.3 \%$, respectively (both $p<0.01$ ). However, ART treatment did not induce cancer cell apoptosis (Fig. 4A).

\section{ART and DHA on pro-inflammatory cytokine expression}

Previous research has reported that the pro-inflammatory cytokine IL-8 recruited and activated neutrophils, which contribute to the gut mucosal damage. Extract from the plant Artemisia annua showed anti-inflammatory activities in different acute and chronic inflammatory models. To test the antiproliferative potentials of Artemisia annua's major constituent and its metabolite, in this study, the anti- 
inflammatory effects of ART and DHA on LPS-induced IL-8 secretion in HT-29 cells were investigated. As shown in Fig. 4B, both ART and DHA significantly inhibited IL-8 secretion at the concentrations of 4-10 uM (both $p<0.01$ ). Our data suggests that both ART and DHA have similar anti-inflammatory potential.

\section{Effects of ART on Treg, Th1 and Th17 Cell Differentiation}

In our previous anti-inflammatory cytokine expression investigation, both ART and DHA showed obvious inhibitory effects on IL-8 secretion. To compare the effects with DHA, the effects of ART on T cell differentiation were assayed. As shown in Fig. 5, ART did not influence Treg cell differentiation. However, ART influenced Th1 and Th17 cell differentiation. For the Th1 cells, ART inhibited the cell differentiation at concentrations of $0.5-2 \mu \mathrm{M}$. For Th17 cells, inhibitory effects of ART was observed at the concentrations of 1 and $2 \mu \mathrm{M}$. Therefore, we observed dose-dependent effects of ART on Th1 and Th17 cell differentiation.

\section{DHA Increased Treg Differentiation and Decreased Th17 Differentiation}

To investigate DHA on adaptive immunity, GBC on T cell differentiation was evaluated. Treg cells act as suppressor cells with unique immune regulation. The normality of Treg cells plays crucial role in the maintenance of the body's immune tolerance. It has been shown that suppression of Th17-cell differentiation from naive $C D 4^{+} T$ cells has beneficial effects on inflammatory disease management. To test DHA on the differentiation of Treg, Th1, and Th17, CD 4 ${ }^{+}$T cells were maintained under Treg, Th1, and Th17-polarizing conditions. At the treatment concentrations of $0.5 \mu \mathrm{M}$, DHA did not influence Treg, Th1, and Th17 cell differentiation obviously. For Th1 cells, only $2 \mu \mathrm{M}$ of DHA showed some effects. For the Treg cells, interestingly, 1 and $2 \mu \mathrm{M}$ of DHA significantly increased Treg cell differentiation (both $p<0.01$ ). However, for the Th17 cell differentiation, approximately $30.4 \%$ of $\mathrm{CD} 4^{+} \mathrm{T}$ cells were IL-17 ${ }^{+}$in the control group. $1 \mu \mathrm{M}$ of DHA treatment inhibited Th17 differentiation to $22.1 \%(p<0.01)$. Further, $2 \mu \mathrm{M}$ of DHA treatment inhibited Th17 differentiation down to $9.5 \%(p<0.01)$ (Fig. 6). Concentration-dependent positive regulation in Treg and negative regulation in Th17 differentiation by DHA treatment was observed.

\section{Discussion}

Colorectal cancer (CRC) remains a leading cause of morbidity and mortality worldwide (Siegel et al., 2021). In spite of the continual advances in developing treatment strategies for CRC patients, doselimiting toxicity and other severe side effects remain as limitations for chemotherapies. The consequences of the drugs can end in refusal to continue with chemotherapy (Schuurhuizen et al., 2017); Cancer patients often try to treat its side effects through complementary and alternative medicinal means. Furthermore, botanical extracts enhance chemopreventive effects of chemotherapeutic agents. As a tremendous chemical diversity is found in millions of species of plants, botanicals, their extracts, and single compounds have been a valuable source of new therapeutic candidate compounds for CRC management. 
Artemisia annua L. is an aromatic annual herbaceous plant. In oriental countries, this herbal medicine traditionally used for fever, inflammation, and malaria-related symptoms $[1,28]$. Phytochemical studies have identified various constituents from this herb including terpenoids, steroids, coumarins, phenolics, flavonoids, and purines, etc [29]. ART is a sesquiterpene lactone isolated from the aerial parts of $A$. annua. In addition to antimalaria action, since ART is a hydrophobic compound thus capable of permeating through the cellular membrane to elicit anti-cancer functions. Although ART's anticancer activities have been reported, its effects were relatively unimpressive [19, 20].

Dihydroartemisinin (DHA) is a metabolite of artemisinin $[6,7]$. To date, many artemisinin derivatives, including its metabolite DHA, have been evaluated for variable medical conditions, including inflammation and malignancies. Compared to ART, DHA has a stronger anti-malaria activity, and has the advantages of better water solubility. Together with its established safety record in anti-malarial treatment, DHA possesses promising drug profiles in the crossover usage as a potential anticancer agent. However, previous studies focus more on direct cancer cell inhibition mechanisms. The impact of inflammation in cancer development, especially the link of adaptive immune response in cancer chemoprevention were neglected. Further, published reports focused on single compound pharmacology, no bioactive comparison between ART and DHA has been performed.

To qualitative and quantitative determination of ART and its metabolites, an HPLC method was developed to determine the purity of ART and DHA. Literature reported method for the determination of ART and DHA are based on WHO criteria, in which a isocratic mobile phase is used, with the composition of acetonitrile:water 60:40 [25], and containing buffer to increase the separate resolution [26, 27]. Based on our observations, ART and DHA $\beta$ could not be separated with the isocratic mobile phase. In this study, a gradient elution program without buffer was developed, with which ART and DHA are baseline separated and they can be determined accurately. The purities of both the two compounds are over $98 \%$, suggesting the high purity of the compound used in this study.

Although DHA is a metabolite of ART, whether the metabolism is mainly after its gastrointestinal absorption or mediated via the biotransformation of gut microbiome is not clear. In this study, gut microbial biotransformation of ART was conducted. Due to the thermostability issues of ART, the temperature during sample preparation needs to be controlled strictly. Thus, $37^{\circ} \mathrm{C}$ was selected to evaporate solvent. Based on previous reports, ethyl acetate was used to extract ART and DHA from the biological fluid [7]. However, because of the high boiling point of ethyl acetate, after extraction, overnight waiting was needed to remove ethyl acetate. Since ART and DHA easily dissolved in ethyl ether, ethyl ether was selected to extract ART and its metabolites. After extraction, ethyl ether could be evaporated on $37^{\circ} \mathrm{C}$ water bath in only $20 \mathrm{~min}$. Therefore, our method saved time, significantly increased the efficacy of sample processing.

Up to date, there is no enteric microbiota biotransformation report on ART. Our gut microbiome transformation results showed that ART was completely converted to its metabolites in $24 \mathrm{~h}$, but DHA is not an ART's gut microbial metabolite. Although DHA was not detected in ART's reaction solution, for the 
first time, our results suggested that ART can be biotransformed by gut microbiome in $24 \mathrm{~h}$, and one metabolite was detected. The whole picture of identification of gut microbial metabolites of the ART should be is an important task in the future.

Using MTS assay, antiproliferative effects of ART and DHA were evaluated. DHA showed stronger antiCRC cell activities than ART. The analysis on cell cycle distribution demonstrated that DHA targeted cell cycle checkpoint for G2/M arrest. Ayclin A plays an important role in controlling the $\mathrm{G} 2 / \mathrm{M}$ transition of the cell cycle by binding to CDK2 $[30,31]$. Our results showed that DHA up-regulated cyclin A, indicating that DHA could facilitate the cell's progression through the $S$ phase into the $\mathrm{G} 2 / \mathrm{M}$ phase and lead to the accumulation of cell distribution in the G2/M phase. Apoptosis is caspase dependent cell death, which is considered an important pathway in the inhibition of cancer cells by many anticancer agents [32]. Caspases are a family of cysteine proteases that play essential roles in cell apoptosis [33]. DHA induced cancer cell apoptosis, suggesting that antiproliferation of DHA on human colorectal cancer cells is mediated in part by the induction of apoptosis (Fig. 7).

Since IBD and chronic inflammation is recognized as a risk factor for CRC development [34], an effective prevention method of malignant colon tumor formation and progression is targeting inflammatory pathways [35]. The adaptive immune response has been considered to play a critical role in the pathogenesis of IBD. T lymphocytes represent the key cell population of the adaptive immunity arm [36]. The original T cells amplify and are differentiated into different subsets such as Th1, Th17, and Treg cells after stimulation of antigens. Th1 cells eliminate pathogens inside cells; Th17 cells remove extracellular bacteria and fungi; Treg cells promote tissue repairs. However, disorders of T cell responses and imbalance of $T$ cell subsets induce excessive releases of cytokines and chemokines, which lead to inflammation [36]. To explore ART and DHA on adaptive immune responses, the effects of those two compounds on the differentiation of Th1, Th17, and Treg cells have been investigated.

Based on our results, although ART did not change Treg cell differentiation, it significantly inhibited Th1 and Th17 cell differentiation. On the other hand, DHA treatment moderately down-regulated Th1 cell differentiation. Interesting, DHA significantly increased Treg differentiation and decreased Th17 differentiation in a dose-dependent manner. Compared to ART, DHA showed more potent inhibitory effects on Th17 than that of ART. Our data suggested that DHA promote Treg differentiation and suppressed differentiation of Th17 cells, which are two important Th subset connects to gut inflammation.

Activated Th17 cells mainly function through secreting cytokines, such as IL-17, IL-21, and IL-22. IL-17 induces immune cells transferring into peripheral tissues and binding with IL-17 receptors, and then activates NF-KB and promotes releases of a variety of pro-inflammatory factors. Treg cells inhibit other Th cells such as Th1 and Th17 via direct contacts with cells and releases of cytokines such as IL-10 and TGF- $\beta$ to maintain immune tolerances. Th17 cells and Treg cells are in dynamic equilibrium under normal circumstances. The balance is broken with over-increases of Th17 cells, over-enhancements of immunogenicity and decreases or abnormal functions of Treg cells, causing intestinal mucosal damages. 
Based on our data, DHA increased Treg differentiation and inhibited Th17 differentiation (Fig. 7). The ratio of Th17/Treg in IBD patients' blood is significantly higher, suggesting that transformation imbalance of Th17/Treg plays an important role on pathogenesis of IBD. Therefore, regulation of balance between Th17/Treg become a new approach for IBD management [37]. Our data suggested that DHA may have the potential to regulate the balance of Th17/Treg, to inhibit gut inflammation and to prevent colon cancer initiation and development.

\section{Conclusion}

In this study, a HPLC method for ART and DHA identification was developed, and accompanying this method with ethyl ether extraction significantly increased the efficacy of sample processing. Gut microbiome biotransformation of ART was performed, and, for the first time, ART was observed to be completely converted to its metabolites within $24 \mathrm{~h}$, but no DHA was detected as a gut microbial metabolite of ART. Using human colorectal cancer cells, ART did not influence cancer cell growth in the tested concentrations but, DHA significantly inhibited the cancer cell growth. The DHA induced G2/M cell cycle arrest via upregulation of cyclin A and apoptosis. As a metabolite of ART, DHA also downregulated the expressions of pro-inflammatory cytokine IL-8. Further, DHA promote Treg cell proliferation, while both ART and DHA inhibited Th1 and Th17 cell differentiation. Our results suggest that DHA is an active anticancer metabolite of ART. While more DHA research should be conducted on cancer chemoprevention, derivatives including novel DHA metal complex should be considered via chemical synthesis, since these complexes have been proven to be effective in cancer therapeutics.

\section{Declarations}

\section{Data availability}

The analyzed data sets generated during the study are available from the corresponding author on reasonable request.

\section{Acknowledgements}

This work was supported in part by the NIH/NCCAM grants P01 AT004418, K01 AT005362, and 5P30DK042086.

\section{Author information}

Chong-Zhi Wang and Chunping Wan are co-first authors, who contribute equally to this study.

\section{Affiliations}

Central Laboratory, the No. 1 Affiliated Hospital of Yunnan University of Traditional Chinese Medicine, Kunming 650021, China 
Tang Center for Herbal Medicine Research, and Department of Anesthesia \& Critical Care, University of Chicago, Chicago, IL 60637, USA

Chong-Zhi Wang, Chunping Wan, Yun Luo, Zhi Liu, Daniel Henry Wang, Mallory Lager \& Chun-Su Yuan

School of Traditional Chinese Pharmacy, China Pharmaceutical University, Nanjing, Jiangsu 210009, China

Chun-Feng Zhang

School of Chemistry and Chemical Engineering, Chongqing University, Chongqing 400044, China Qi-Hui Zhang

School of Pharmacy, Nanjing Medical University, Nanjing, Jiangsu 211166, China

Lina Chen

Tang Center for Traditional Chinese Medicine Research, Institute of Chinese Materia Medica, China Academy of Chinese Medical Sciences, Beijing 100700, China

Cang-Hai Li \& Ting-Liang Jiang

Program in Cellular and Molecular Medicine, Boston Children's Hospital and Department of Pediatrics, Harvard Medical School, Boston, MA 02115, USA

Lifei Hou

Committee on Clinical Pharmacology and Pharmacogenomics, University of Chicago, Chicago, IL 60637, USA

Chun-Su Yuan

Contributions

Project administration: CZW, CSY. Participated in research design: CFZ, QHZ, LC, ZL, LH. Conducted experiment: CZW, CW, YL, DHW. Wrote an original draft preparation: CZW, ML, CSY. Performed data analysis: CW, YL. Edited and reviewed: CZW, CHL, TLJ, CSY.

Corresponding author

Correspondence to Chong-Zhi Wang

Ethics declarations 


\section{Conflict of interest}

The authors declare that there are no conflicts of interest.

\section{Consent to participate}

All authors have agreed to participate in the manuscript.

\section{Consent for publication}

All authors have agreed to publish the manuscript.

\section{Ethical approval}

All procedures for experimental protocols of the present study involving animals and cells were performed in accordance with the ethical standards of the institution of practice at which the studies were conducted.

The need for ethics approval for human fecal related study was waived by the local medical ethics committee and is deemed unnecessary according to local guidelines. It did not pertain with any (clinical/biological) investigation of any human tissue at any level. Verbal and written consent was obtained from all participants. Ethics approval was not required by the local medical ethics committee as it was deemed unnecessary according to committee's requirements.

\section{References}

1. van der Kooy F, Sullivan SE (2013) The complexity of medicinal plants: the traditional Artemisia annua formulation, current status and future perspectives. J Ethnopharmacol 150:1-13.

2. Tu Y (2016) Artemisinin-A Gift from Traditional Chinese Medicine to the World (Nobel Lecture). Angew Chem Int Ed Engl 55:10210-10226.

3. Fan TT, Cheng BL, Fang XM, Chen YC, Su F (2020) Application of Chinese Medicine in the Management of Critical Conditions: A Review on Sepsis. Am J Chin Med 48:1315-1330.

4. Su XZ, Miller LH (2015) The discovery of artemisinin and the Nobel Prize in Physiology or Medicine. Sci China Life Sci 58:1175-1179.

5. Wang CZ, Zhang Z, Wan JY, Zhang CF, Anderson S, He X, et al (2015) Protopanaxadiol, an active ginseng metabolite, significantly enhances the effects of fluorouracil on colon cancer. Nutrients 7:799-814.

6. Edikpo NJ, Adikwu E (2013) Artemisinin: an evolving antimalarial-part one. Br J Pharmacol Toxicol 4:241-255.

7. Ma Y, Sun P, Zhao Y, Wang K, Chang X, Bai Y, et al (2019) A microbial transformation model for simulating mammal metabolism of artemisinin. Molecules 24:315. 
8. Hu D, Wang Y, Chen Z, Ma Z, You Q, Zhang X, et al (2014) Artemisinin protects against dextran sulfate-sodium-induced inflammatory bowel disease, which is associated with activation of the pregnane X receptor. Eur J Pharmacol 738:273-284.

9. Yan YX, Shao MJ, Qi Q, Xu YS, Yang XQ, Zhu FH, et al (2018) Artemisinin analogue SM934 ameliorates DSS-induced mouse ulcerative colitis via suppressing neutrophils and macrophages. Acta Pharmacol Sin 39:1633-1644.

10. Li N, Sun W, Zhou X, Gong H, Chen Y, Chen D, et al (2019) Dihydroartemisinin protects against dextran sulfate sodium-induced colitis in mice through inhibiting the PI3K/AKT and NF-kappaB signaling pathways. Biomed Res Int 2019:1415809.

11. McCarthy N (2013) Tumorigenesis: All together now. Nat Rev Cancer 13:148-149.

12. Al Bakir I, Curtius K, Graham TA (2018) From colitis to cancer: An evolutionary trajectory that merges maths and biology. Front Immunol 9:2368.

13. Lian F, Li G, Chen X, Wang X, Piao C, Wang J, et al (2014) Chinese herbal medicine Tianqi reduces progression from impaired glucose tolerance to diabetes: a double-blind, randomized, placebocontrolled, multicenter trial. J Clin Endocrinol Metab 99:648-655.

14. Poudyal D, Le PM, Davis T, Hofseth AB, Chumanevich A, Chumanevich AA, et al (2012) A hexane fraction of American ginseng suppresses mouse colitis and associated colon cancer: antiinflammatory and proapoptotic mechanisms. Cancer Prev Res (Phila) 5:685-696.

15. Madka V, Rao CV (2013) Anti-inflammatory phytochemicals for chemoprevention of colon cancer. Curr Cancer Drug Targets 13:542-557.

16. Ahangarpour A, Sayahi M (2019) The antidiabetic and antioxidant properties of some phenolic phytochemicals: A review study. Diabetes Metab Syndr 13:854-857.

17. Efferth T (2017) From ancient herb to modern drug: Artemisia annua and artemisinin for cancer therapy. Semin Cancer Biol 46:65-83.

18. Zhao Y, Niu Y, He J, Gan Z, Ji S, Zhang L, et al (2020) Effects of dietary dihydroartemisinin supplementation on growth performance, hepatic inflammation, and lipid metabolism in weaned piglets with intrauterine growth retardation. Anim Sci J 91:e13363.

19. Kim EJ, Kim GT, Kim BM, Lim EG, Kim SY, Kim YM (2017) Apoptosis-induced effects of extract from Artemisia annua Linne by modulating PTEN/p53/PDK1/Akt/ signal pathways through PTEN/p53independent manner in HCT116 colon cancer cells. BMC Complement Altern Med 17:236.

20. Ho WE, Peh HY, Chan TK, Wong WS (2014) Artemisinins: pharmacological actions beyond antimalarial. Pharmacol Ther 142:126-39.

21. Du GJ, Zhang Z, Wen XD, Yu C, Calway T, Yuan CS, et al (2012) Epigallocatechin gallate (EGCG) is the most effective cancer chemopreventive polyphenol in green tea. Nutrients 4:1679-1691.

22. Wang CZ, Zhang Z, Huang WH, Du GJ, Wen XD, Calway T, et al (2013) Identification of potential anticancer compounds from Oplopanax horridus. Phytomedicine 20:999-1006. 
23. Hou L, Cooley J, Swanson R, Ong PC, Pike RN, Bogyo M, et al (2015) The protease cathepsin L regulates Th17 cell differentiation. J Autoimmun 65:56-63.

24. Wang CZ, Hou L, Wan JY, Yao H, Yuan J, Zeng J, et al (2020) Ginseng berry polysaccharides on inflammation-associated colon cancer: inhibiting T-cell differentiation, promoting apoptosis, and enhancing the effects of 5-fluorouracil. J Ginseng Res 44:282-290.

25. Cabri W, Ciogli A, D'Acquarica I, Di Mattia M, Galletti B, Gasparrini F, et al (2008) On-column epimerization of dihydroartemisinin: an effective analytical approach to overcome the shortcomings of the International Pharmacopoeia monograph. J Chromatogr B Analyt Technol Biomed Life Sci 875:180-91.

26. Wang Y, Liu J-K (2012) Improvement of the HPLC determination condition for artemisinin and its derivatives. J Liq Chromatogr Relat Technol 35:1712-1718.

27. Wang L-z, Wu H-d, Liu L, Liu H-z, Liu T-s (2015) Simultaneous determination of artemisinin, dihydroartemisinin and $\beta$-artemether in the synthetic liquid of artemether by HPLC. Zhongchengyao 37:2216-2219.

28. Feng X, Cao S, Qiu F, Zhang B (2020) Traditional application and modern pharmacological research of Artemisia annua L. Pharmacol Ther 216:107650.

29. Brown GD (2010) The biosynthesis of artemisinin (Qinghaosu) and the phytochemistry of Artemisia annua L. (Qinghao). Molecules 15:7603-7698.

30. Hartwell LH, Weinert TA (1989) Checkpoints: controls that ensure the order of cell cycle events. Science 246:629-634.

31. Yamada T, Das Gupta TK, Beattie CW (2016) p28-Mediated activation of p53 in G2-M phase of the cell cycle enhances the efficacy of DNA damaging and antimitotic chemotherapy. Cancer Res 76:2354-2365.

32. Millimouno FM, Dong J, Yang L, Li J, Li X (2014) Targeting apoptosis pathways in cancer and perspectives with natural compounds from mother nature. Cancer Prev Res (Phila) 7:1081-1107.

33. Medina CB, Mehrotra P, Arandjelovic S, Perrys JSA, Guo YZ, Morioka S, et al (2020) Metabolites released from apoptotic cells act as tissue messengers. Nature 580:130-135.

34. El-Dallal M, Chen Y, Lin Q, Rakowsky S, Sattler L, Foromera J, et al (2020) Meta-analysis of virtualbased chromoendoscopy compared with dye-spraying chromoendoscopy standard and highdefinition white light endoscopy in patients with inflammatory bowel disease at increased risk of colon cancer. Inflamm Bowel Dis 26:1319-1329.

35. Ghanghas P, Jain S, Rana C, Sanyal SN (2016) Chemopreventive action of non-steroidal antiinflammatory drugs on the inflammatory pathways in colon cancer. Biomed Pharmacother 78:239247.

36. Huang Y, Chen Z (2016) Inflammatory bowel disease related innate immunity and adaptive immunity. Am J Transl Res 8:2490-2497.

37. Ueno A, Jeffery L, Kobayashi T, Hibi T, Ghosh S, Jijon H (2018) Th17 plasticity and its relevance to inflammatory bowel disease. J Autoimmun 87:38-49. 
A

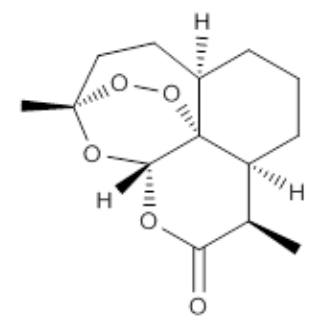

Artemisinin

(ART)

B
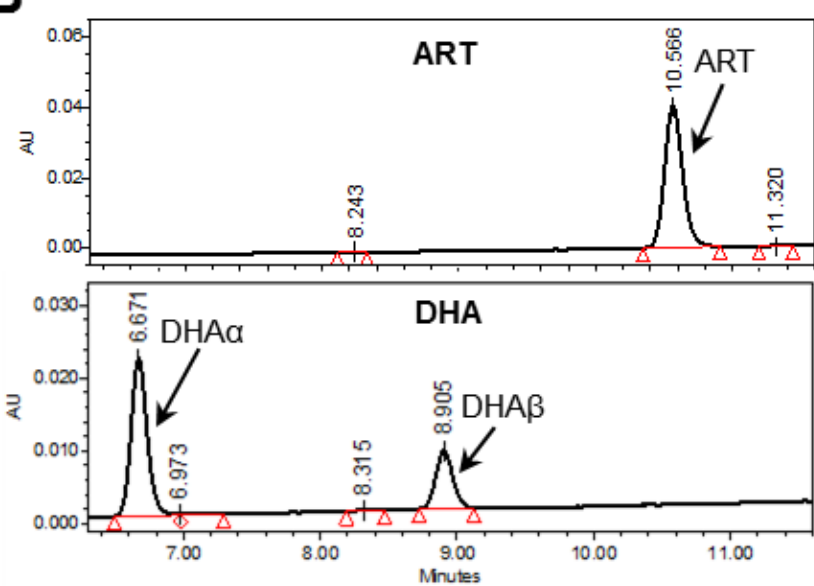

C
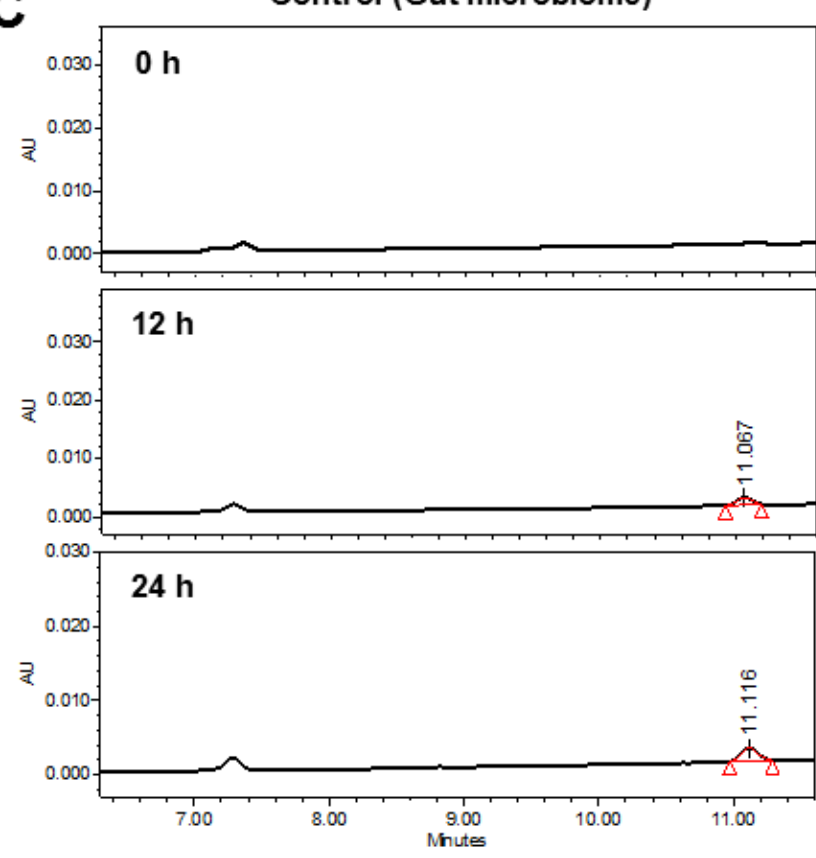

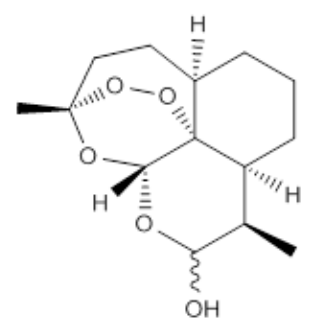

Dihydroartemisinin

(DHA)

UV spectrum
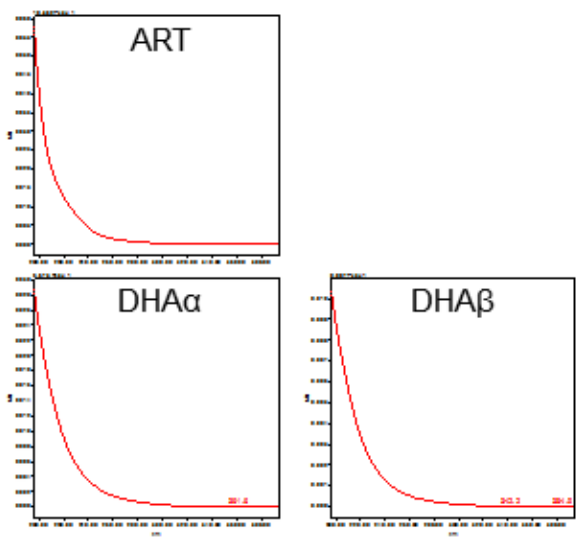

ART + Gut microbiome
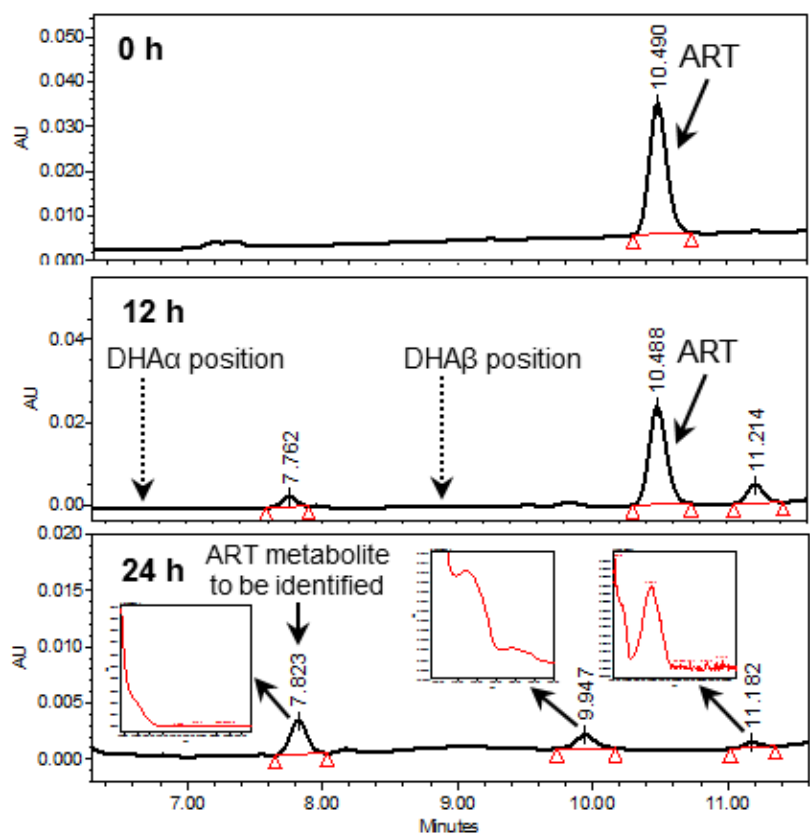

Figure 1

Chemical structures of artemisinin (ART) and dihydroartemisinin (DHA), and gut microbiome transformation of ART. (A) The in vivo metabolism from ART to DHA. (B) Purity determination of ART and DHA. HPLC chromatograms record at $210 \mathrm{~nm}$ and UV spectra from $196 \mathrm{~nm}$ to $400 \mathrm{~nm}$ are presented. (C) 
Gut microbiome biotransformation of ART. Chromatograms for gut microbiome control and ART incubated at $37^{\circ} \mathrm{C}$ for 0,12 and $24 \mathrm{~h}$ are presented. ART is absolutely converted to its metabolites in $24 \mathrm{~h}$, including a compound at Rt 7.8 min, while DHA is not detected. The UV spectra of the ART metabolite (7.8 $\mathrm{min}$ ) and two gut microbiome metabolites (9.9 and $11.2 \mathrm{~min}$ ) are presented.
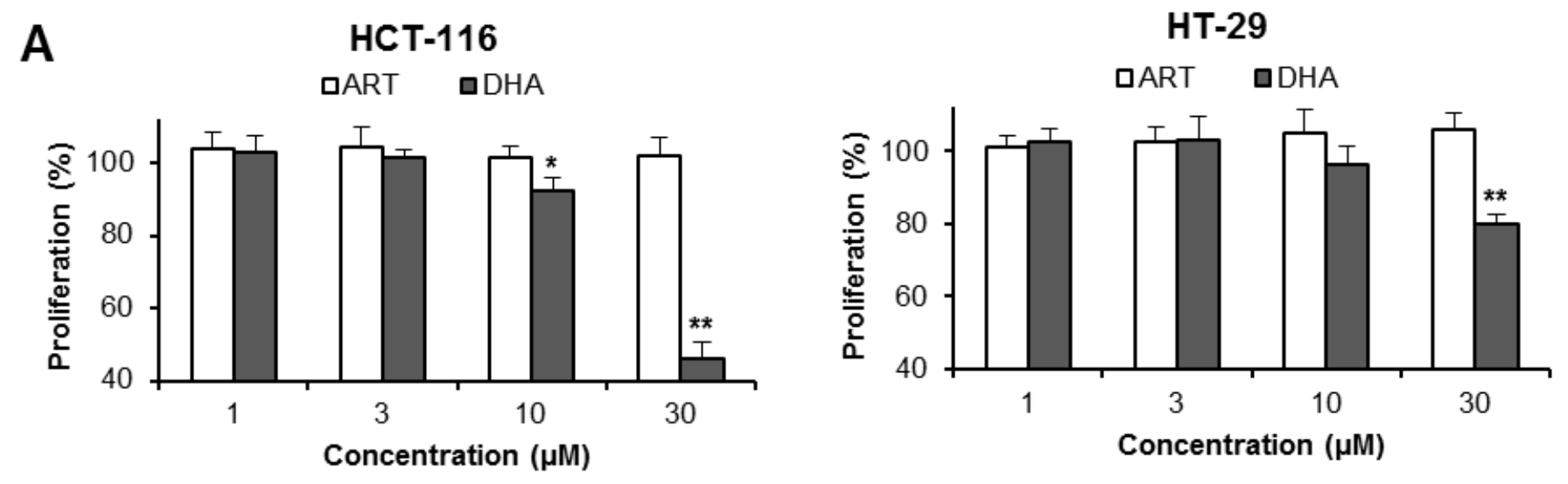

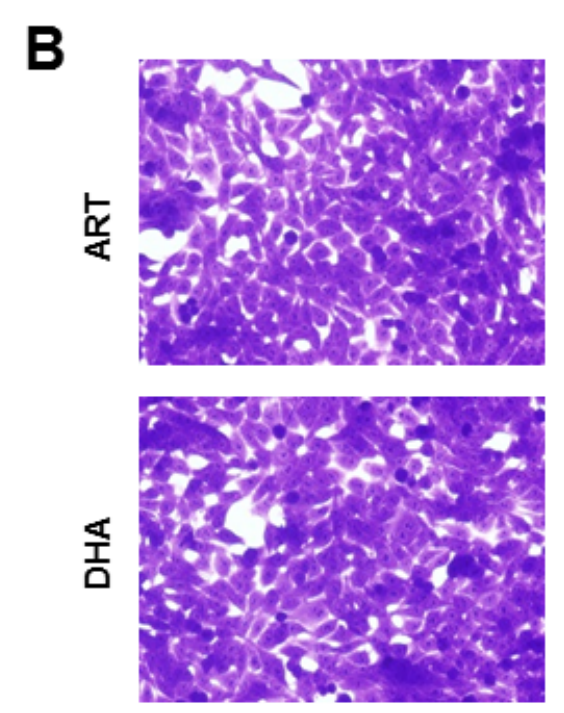

$0 \mu \mathrm{M}$

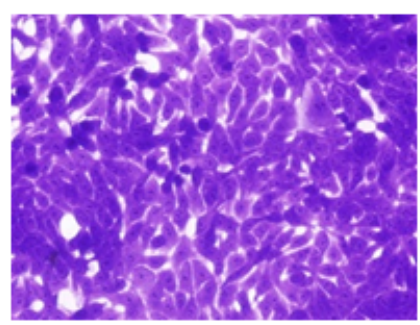

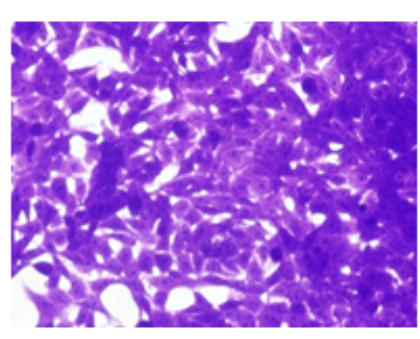

$5 \mu \mathrm{M}$
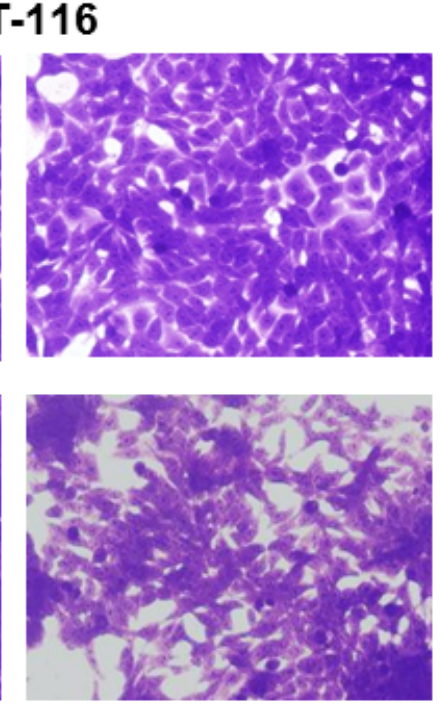

$15 \mu \mathrm{M}$
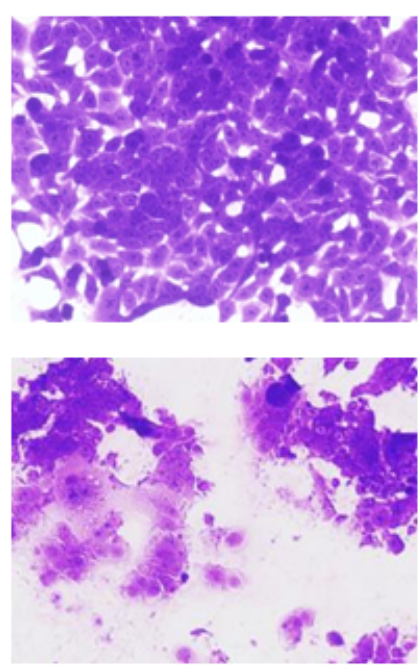

$25 \mu \mathrm{M}$
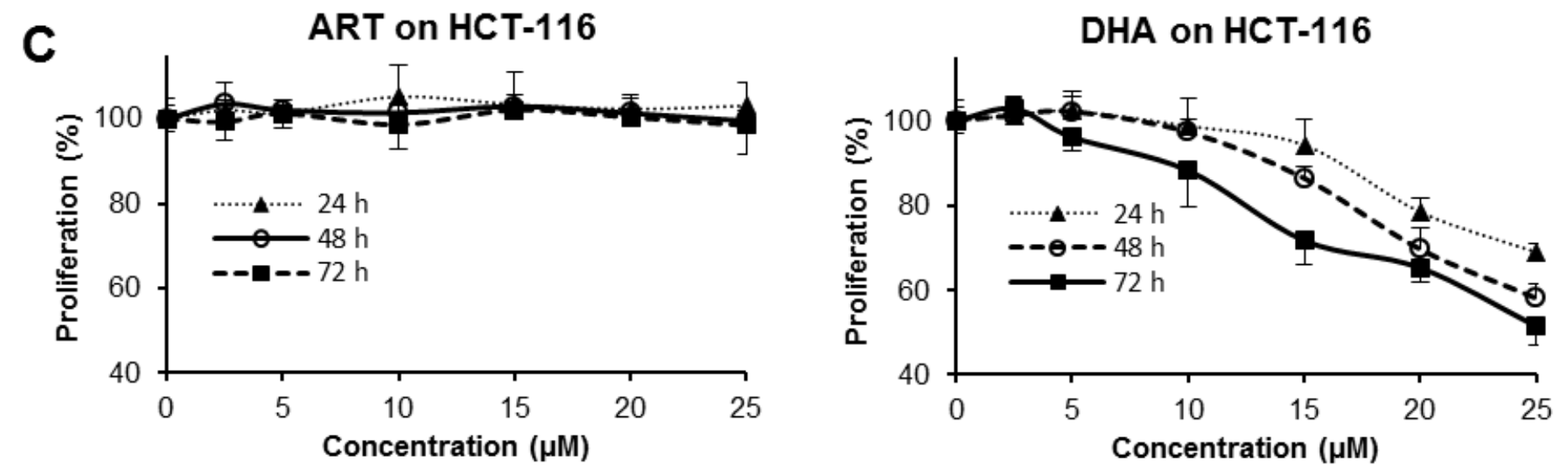

Figure 2 
Antiproliferative effects of ART and DHA on HCT-116 and HT-29 human colorectal cancer cells. (A) Concentration-associated antiproliferative effects on HCT-116 and HT-29 cells for $48 \mathrm{~h}$. Cells were treated with 1-30 $\mu \mathrm{M}$ of tested compounds for $48 \mathrm{~h}$. (B) HCT-116 cells were treated with 5-15 $\mu \mathrm{M}$ of tested compounds for $48 \mathrm{~h}$, and were then stained with crystal purple and photographed showing cell growth inhibition, especially at 15-25 $\mu \mathrm{M}$. (C) Time-associated antiproliferative effects on HCT-116 cells. Cells were treated with $2.5-25 \mu \mathrm{M}$ of tested compounds for 24,48 and $72 \mathrm{~h}$. ${ }^{*} \mathrm{P}<0.05$, ${ }^{*} \mathrm{P}<0.01 \mathrm{vs}$. control.
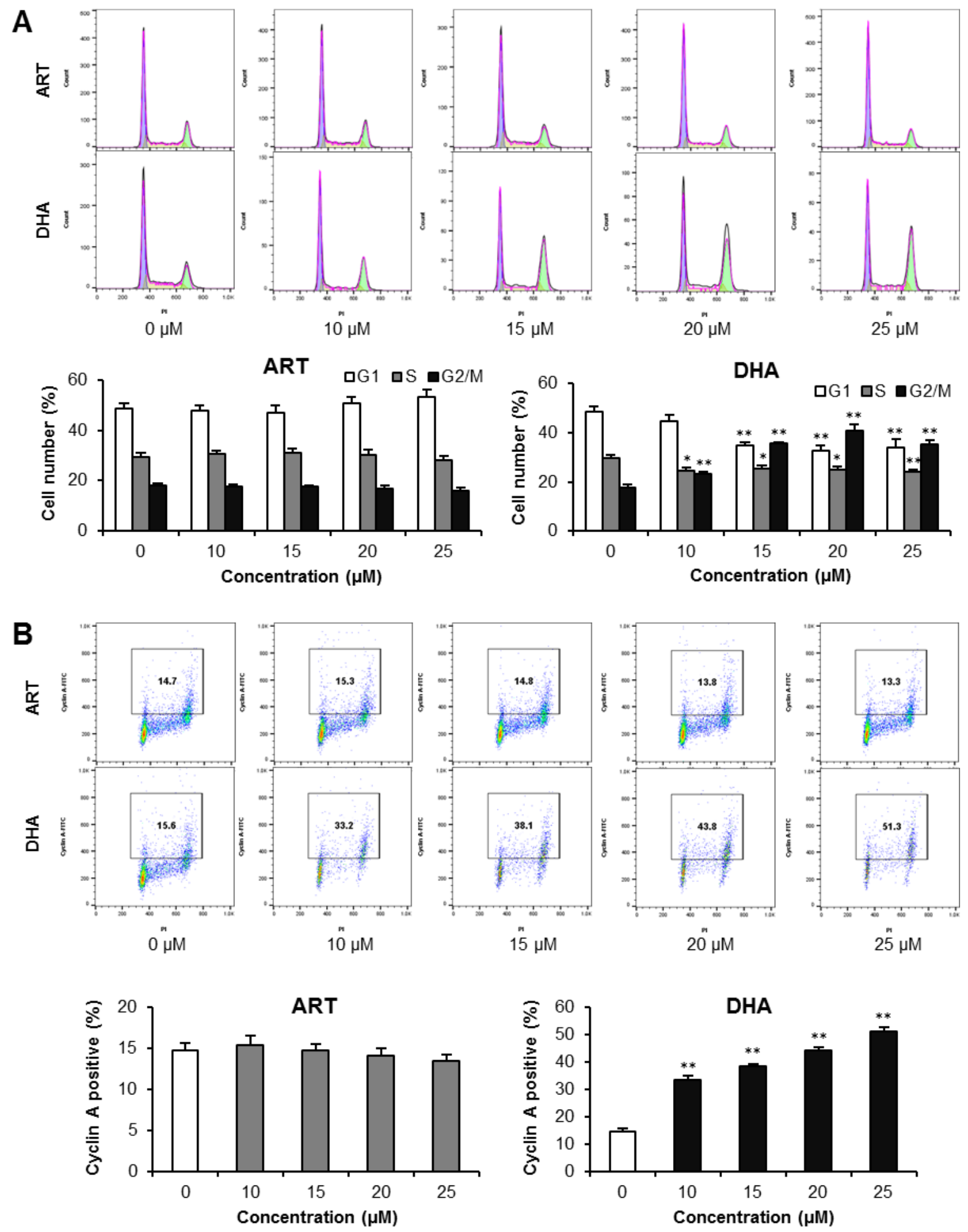

Figure 3 
Effects of ART and DHA on cell cycle arrest and the expression of cyclin HCT-116 cells were treated with $10,15,20$ and $25 \mathrm{uM}$ of ART or DHA for $48 \mathrm{~h}$. The cells were collected and stained with PI/RNase and cyclin A-FITC followed by flow cytometric analysis. (A) Upper panel: The representative histograms of DNA content in different experimental groups. Lower panel: Percentage of each cell cycle phase in different groups. (B) Upper panel: The expression of cyclin A. The numbers in the gates are the percentage of cyclin A positive cells. Lower panel: Percentage of cyclin A-positive cells in different groups. Data obtained from triplicate experiments. * $\mathrm{P}<0.05$, $*$ P $<0.01$ compared to control.
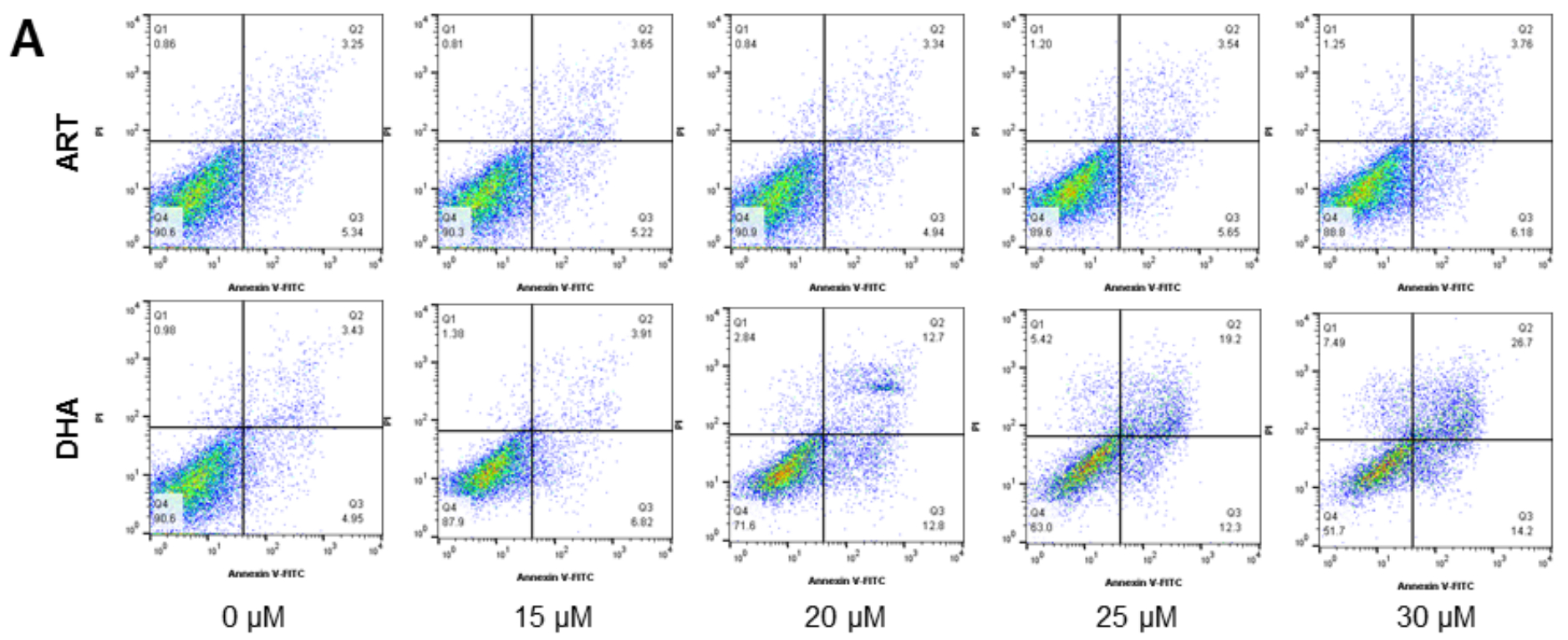

ART
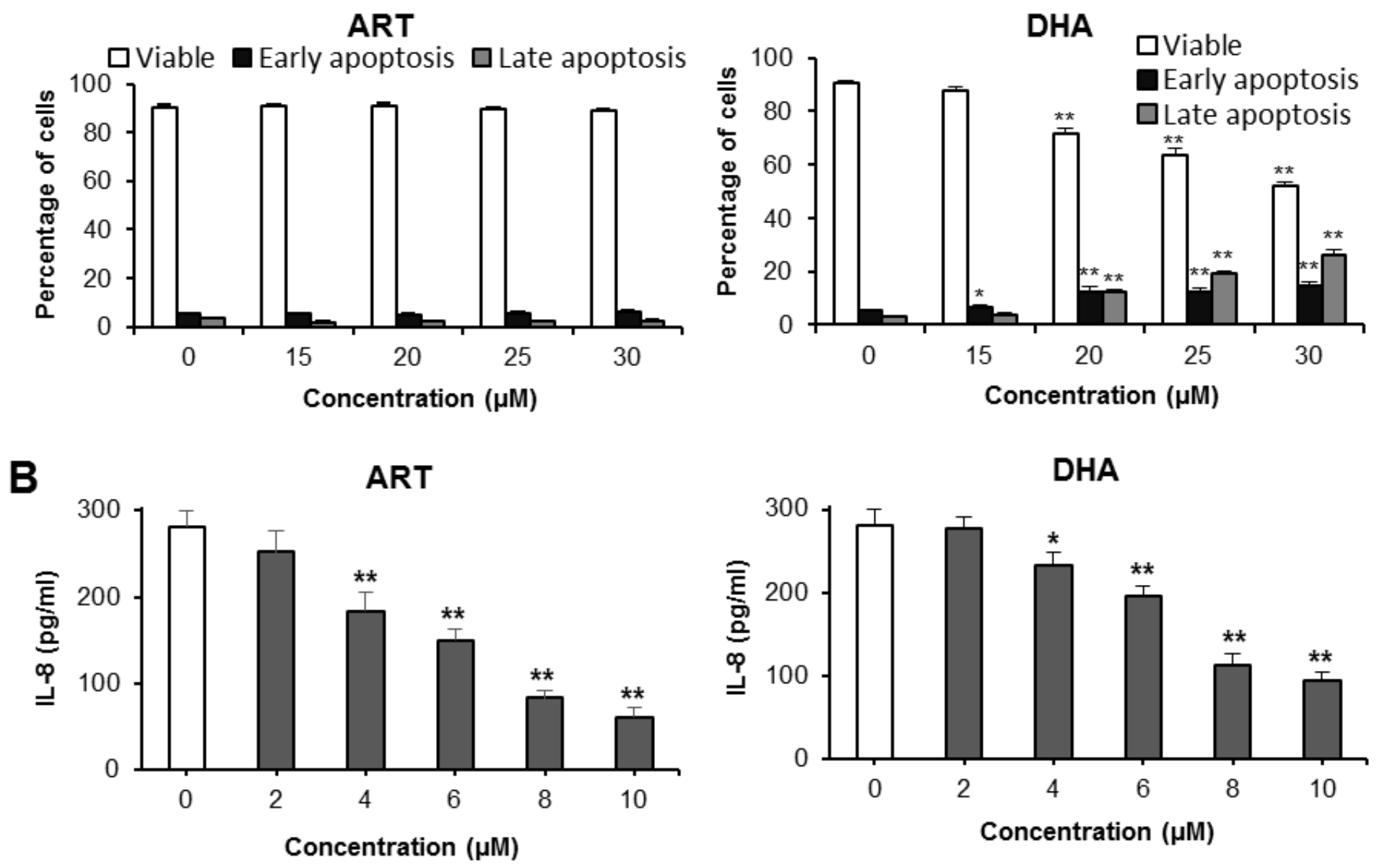


\section{Figure 4}

Apoptotic and anti-inflammatory analyses of HCT-116 cells treated with ART and DHA. (A) HCT-116 cells were treated with $15,20,25$ and $30 \mu \mathrm{M}$ of ART and DHA for $48 \mathrm{~h}$. Apoptosis was quantified using annexin V-FITC/propidium iodide (PI) staining followed by flow cytometric analysis. Upper panel: Representative scatter plots of PI (y-axis) versus annexin V (x-axis). Lower panel: Percentage of viable, early apoptotic and late apoptotic cells. (B) Effects of ART and DHA on inflammatory cytokine IL-8 secretion in HT-29 cells. The basal level of IL-8 secretion from HT-29 cells was very low $(<20 \mathrm{pg} / \mathrm{ml})$. LPS-induced IL-8 secretion was inhibited by both compounds. Data are presented as the mean \pm standard error of triplicate experiments. ${ }^{*} P<0.05, * * P<0.01$ vs. control. 

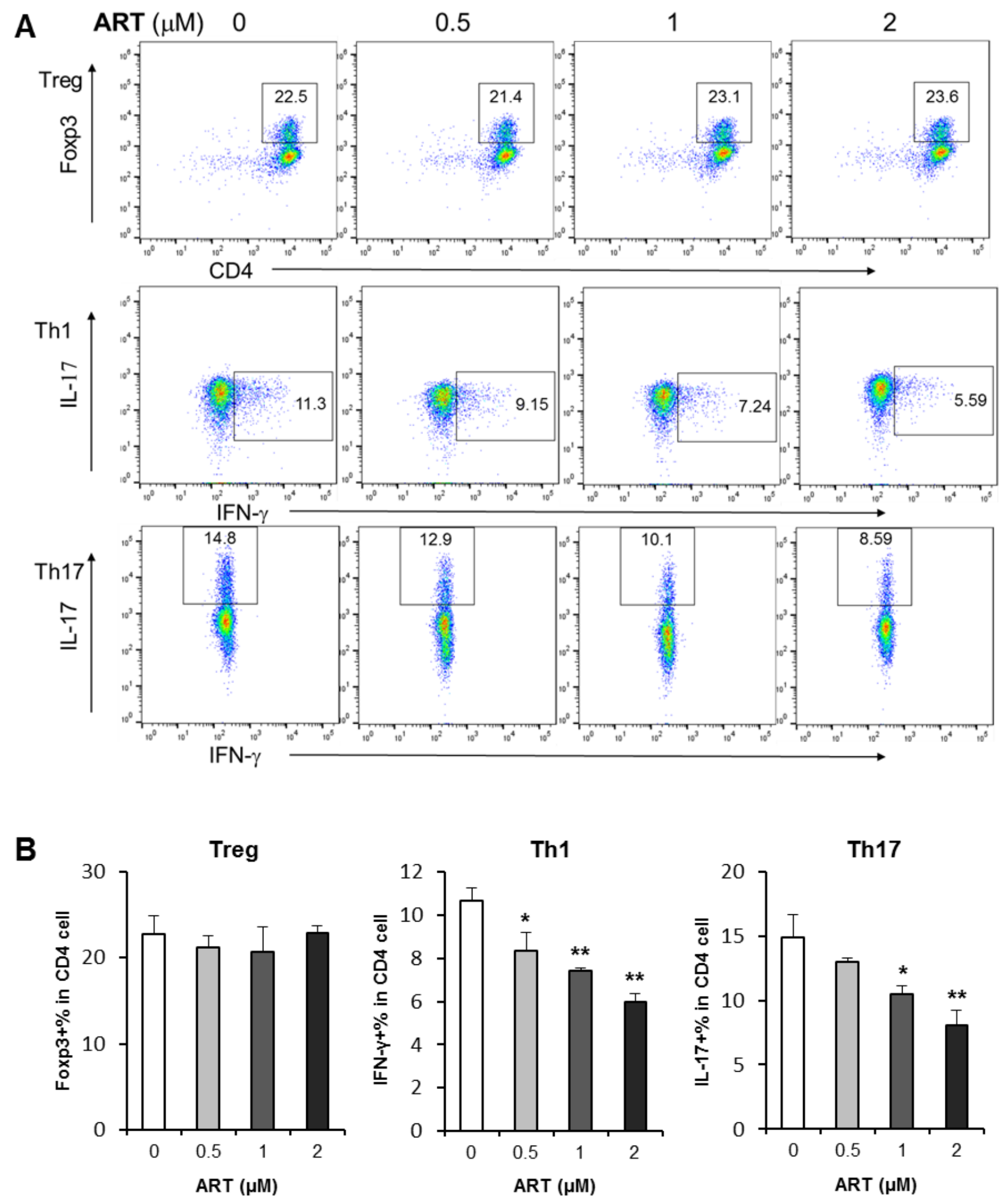

Figure 5

Effect of ART on Treg, Th1, and Th17 cell differentiation. (A) Representative scatter plots assayed by flow cytometry. The numbers in the gates are the percentage of each type of $t$ cells. (B) Data are presented as the mean \pm standard error of triplicate experiments. ${ }^{*} P<0.05,{ }^{*} \mathrm{P}<0.01 \mathrm{vs}$. control. 

A DHA $(\mu \mathrm{M}) \quad 0$

\section{5}
1
2
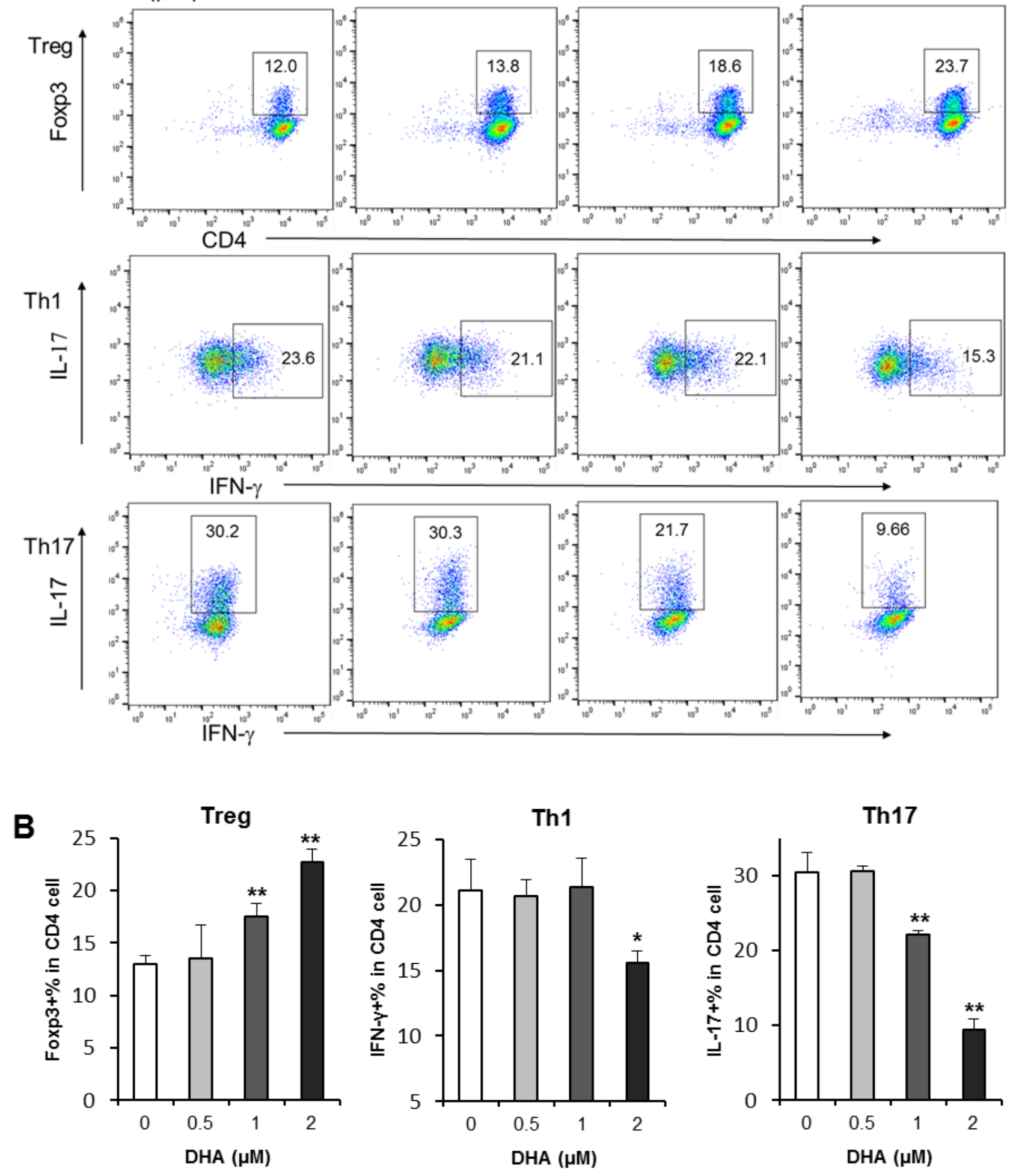

Th17

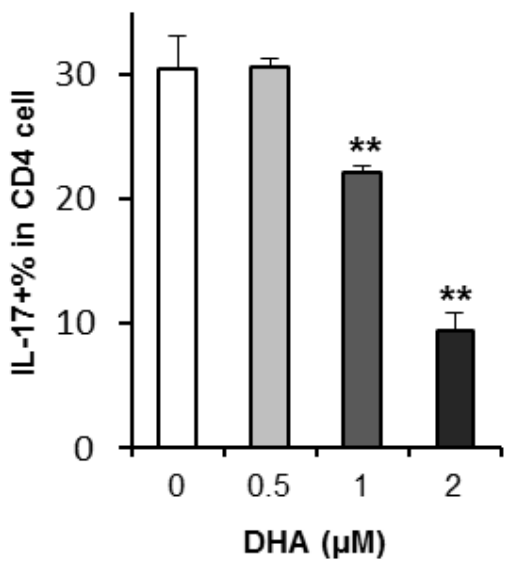

Figure 6

Effect of DHA on Treg, Th1, and Th17 cell differentiation. (A) Representative scatter plots assayed by flow cytometry. The numbers in the gates are the percentage of each type of $t$ cells. (B) Data are presented as the mean \pm standard error of triplicate experiments. ${ }^{*} P<0.05,{ }^{*} \mathrm{P}<0.01 \mathrm{vs}$. control. 


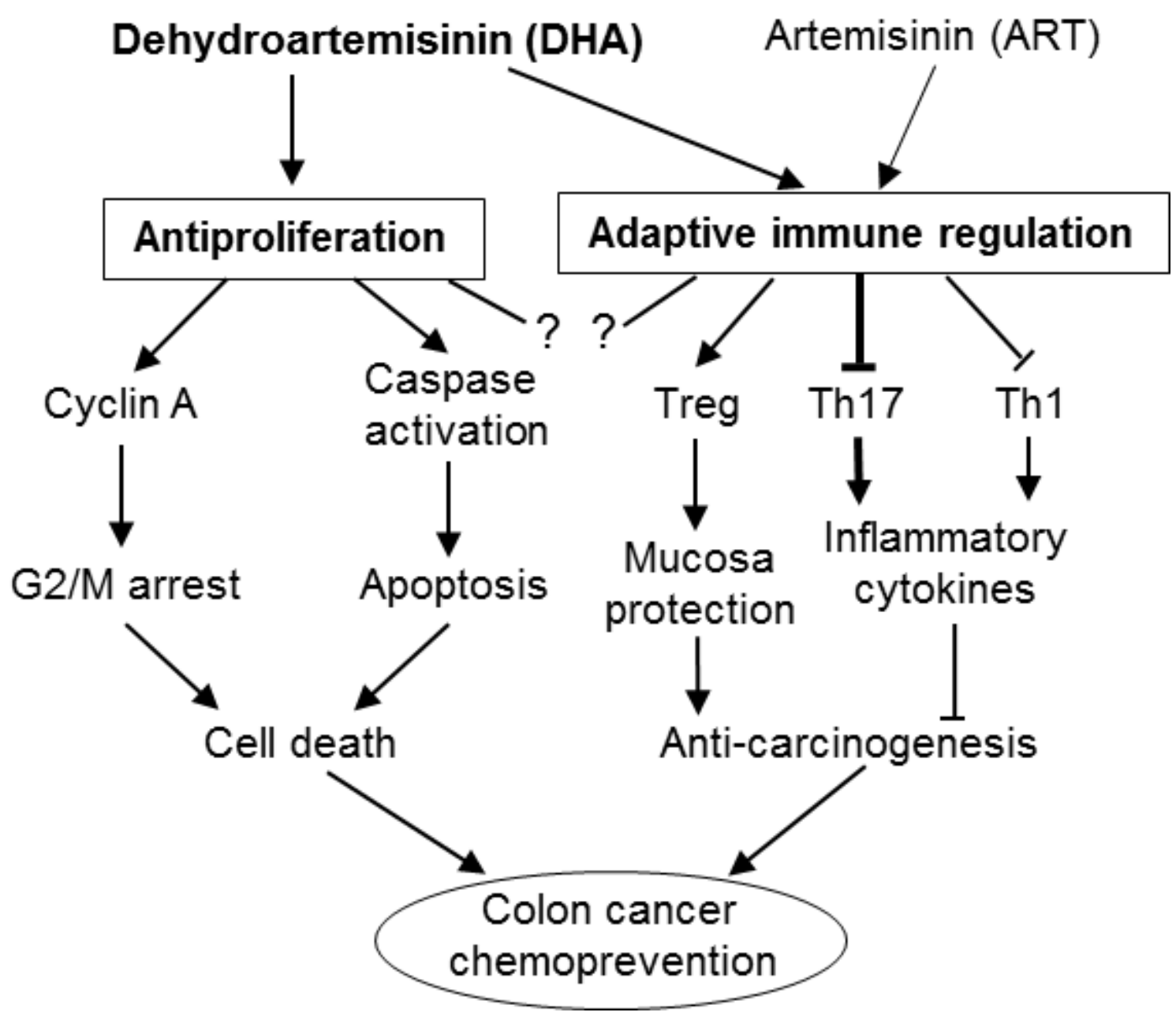

Figure 7

A working model for the actions of DHA, in comparison with ART, on colorectal cancer chemoprevention. DHA induces $\mathrm{G} 2 / \mathrm{M}$ cell cycle arrest and apoptosis in colon cancer cells, which promotes cancer cell death. DHA also protects mucosa cells by up-regulation of Treg cells, and inhibits pro-inflammatory cytokine release by down-regulation of Th1 and Th17 cells, thus inhibited colon cancer carcinogenesis. 